\title{
Los últimos años del período amarniense
}

\author{
ANTONIO PÉrez LARGacha *
}

Referirse al período amarniense, en cualquiera de sus manifestaciones y etapas, implica adentrarse en una polémica constante, con opiniones y teorías contrapuestas en las que tienen no poca incidencia el grado de simpatía o rechazo que la figura de Akhenatón despierta '. El mejor ejemplo de ello son las opiniones antagónicas de dos de los principales especialistas del período, Redford (1984) y Alfred (1988), perfectamente contrapuestas por Eaton-Krauss en su «Akhenaten versus Akhenaten» (1990).

Durante años, el debate ha estado centrado en buscar las razones que llevaron a Amenofis IV (Akhenatón) a llevar a cabo su «reforma», sus años de reinado, las características de su religión y de la ciudad que mandó construir, Akhetaten. Por el contrario, los acontecimientos que siguieron a su muerte, hasta el comienzo de la XIX dinastía, han sido escasamente estudiados, aceptándose a grandes rasgos los planteamientos históricos emitidos a comienzos de nuestro siglo: tras la muerte de Akhenatón, o incluso antes, Egipto emprendió un camino de retorno a la normalidad política y religiosa, con reinados sucesivos de Smenkhare, Tutankhamón, Ay y Horemheb, que se caracterizaron por una restitución en sus cargos y funciones de todas aquellas personas que, por una razón u otra, se habían visto desposeídas de ellos por Akhenatón, al mismo tiempo que la sucesión entre estos faraones fue pacífica y sin convulsiones. Las únicas incógnitas

\footnotetext{
* Universidad de Alcalá de Henares.

1 En las siguientes páginas utilizaremos la transcripción comúnmente utilizada, aun cuando sería más correcto eliminar la partículo $\mathrm{kh}$ por j. Akhenatón = Ajenaton; Tutankhamón = Tutanjamon... Igualmente, nos referiremos a la ciudad que Akhenatón erigió como su capital, bien como Akhetaten, el nombre que recibe en los textos, o como el-Amarna, ya que el término Tell el-Amarna no es correcto, por no presentar la ciudad las características de un Tell habiendo sido acuñado por la existencia de una villa moderna, el-Till, en las cercanías de la ciudad antigua.
} 
que parecían ceñirse sobre este período eran: la petición de Ankhesamón, viuda de Tutankhamón, de un marido al rey Hitita Suppiluliuma y, en segundo lugar, la adscripción original del ajuar funerario de Tutankhamón y de la controvertida tumba KV 55. Estos «dogmas", fijados muchos de ellos a comienzos de nuestro siglo, son difíciles de rebatir o, cuando menos, de matizar, especialmente en lo que concierne a la opinión pública.

Este «olvido» de los años que siguieron al fracaso de Akhenatón y su "revolución» tiene su origen en un error historiográfico presente no sólo en la investigación y comprensión del período amarniense, sino también en otros acontecimientos históricos acaecidos en la Antigüedad: explicar un personaje o etapa de la historia a través de la figura que centra esos años, en nuestro caso, Akhenatón. Sin embargo, al igual que el período amarniense tiene unos antecedentes en la primera mitad de la xvill dinastía, e incluso en el Segundo Período Intermedio (Pérez Largacha 1994), muchos de los aspectos sociales, políticos y de mentalidad presentes en las dinastías XIX-XX, como la piedad personal ramesida, encuentran su explicación, no sólo en la «política revolucionaria» de Akhenatón, sino en la política que tuvieron que llevar a cabo sus inmediatos sucesores con el fin de reestablecer la "normalidad" en el país y justificar sus propios reinados.

Mientras que los primeros años de este fascinante período de la historia de Egipto van reconstruyéndose lentamente gracias al conocimiento y estudio de los talatats (Redford 1977; 1988); la política exterior de Akhenatón va delimitándose de forma que es posible abandonar la idea de un pacifismo religioso (Several 1972; Weinstein 1981; Pérez Largacha 1995), sin que ello implique una activa política militar en el exterior como defiende Schulman (1964, 1979 y 1988); su pretendido monoteísmo puede ser matizado y enmarcado en un programa teológico, religioso y político concreto (Assmann 1983; Allen 1989; Tobin 1986; Mysliwiec 1979 y 1990; Lorton 1993), los últimos años de Akhenatón y su sucesión han comenzado a ser analizados desde otras perspectivas, con la aparición de nuevas hipótesis que matizan la sucesión faraónica propuesta, interpretaciones basadas en nuevos materiales arqueológicos y epigráficos que proporcionan luz, y también sombras, a este período de la historia de Egipto, pudiéndose fijar el comienzo del debate sobre estos años con la aparición del libro de Krauss (1978).

Nuestra intención en las próximas páginas será analizar este período, estudiando a los distintos personajes que lo protagonizan pero, especialmente, emitir una serie de planteamientos que pueden abrir nuevas vías de investigación, pudiendo futuros hallazgos arqueológicos y nuevas lecturas epigráficas modificar, confirmar o matizar lo aquí expuesto. 
Finalmente, antes de adentrarnos en los sucesores de Akhenatón, debemos hacer una breve referencia a los últimos años de su misterioso y fascinante reinado.

\section{Amarna tras el año 12}

El año 12 del reinado de Akhenatón ${ }^{2}$ se ha apuntado como el comienzo del declive de la «herejía» amarniense. La muerte de la reina madre Tiye, de la hija de Akhenatón y Nefertiti, Meketaten, y de la propia Nefertiti según algunos, son acontecimientos que pudieron marcar la personalidad de Akhenatón y un abandono progresivo de sus ideas. Estas muertes de los miembros de la familia real se han puesto en relación con una posible epidemia de peste llegada a Egipto desde la costa sirio-palestina, plaga que el gobernador de Biblos niega ${ }^{3}$, pero que constatamos años más tarde en Hatti (Helck 1978). En relación con dicha epidemia, Aldred (1988: 283) pone en relación las más de 700 estatuas erigidas por Amenofis III a la diosa Sekhmet ${ }^{4}$. Las únicas muertes que con seguridad pueden confirmarse son las de Meketaten, representada en la tumba real de Amarna (Martin 1989a), y la de Kiya, una esposa secundaria de Akhenatón (Reeves 1988), cuya muerte, según Martin (1989), es representada en la tumba real de el-Amarna dando a luz a Tutankhamón.

Aparte de la incidencia que estas muertes pudieran tener en la personalidad de Akhenatón, en ese mismo año 12 tuvo lugar la recepción de tributos y embajadores asiáticos en el-Amarna que, según algunos, no fue sino el comienzo de la pérdida de los intereses egipcios en Siria-Palestina, debido a la dejación de Akhenatón, la actitud del reino de Amurru y la política realizada por Suppiluliuma en el Norte de Siria, declive que se refleja en las cartas del archivo de el-Ámarna ${ }^{5}$.

2 Esta fecha es adoptada sin tener en cuenta si existió una corregencia entre Amenofis III y Amenofis IV (cf., MURNAME 1977 obra que sigue reuniendo todos los materiales susceptibles de apoyar o no dicha corregencia).

3 EA 129a: 45.

4 No hemos de olvidar que ALDRED defiende una corregencia de 12 años entre Amenofis III y su hijo.

5 Sobre Amurru y la política Hitita en el Norte de Siria, cf., Redford 1992; Kitchen 1962; Spalinger 1979, Murname 1990. Respecto al archivo de el-Amarna, su contenido debe utilizarse con cautela (SeVERAL 1972; LiveranI 1983; Pérez LaRGACHA 1994), aunque Schulman (1988) piensa que de algunas cartas se obtiene la impresión de que se estaba preparando una campaña hitita, a pesar de que en algunas cartas los príncipes leales reconocen que Egipto no enviará ayuda. 
En este marco histórico, suele señalarse que poco después del año 12 , o en el mismo, y tras la muerte u ostracismo de Nefertiti, su hija Meritaten fue elevada al cargo y status de Esposa principal, casándose con Smenkhare, faraón de Egipto durante 3 años y posible hermano de Tutankhamón, con quienes la "ortodoxia" comenzó a volver en Egipto. Sin embargo, emergen distintas incógnitas; ¿existió un faraón entre Akhenatón y Smenkhare?, ¿llegó Nefertiti a gobernar Egipto en los últimos años de existencia de Akhetaten?, ¿hubo una corregencia entre Akhenatón y Smenkhare?, ¿comenzó Smenkhare el acercamiento al clero de Amón y a las tradicionales familias nobiliarias desplazadas por el movimiento amarniense? ${ }^{6}$, ¿fueron Smenkhare y Tutankhamón hermanos?, ¿intentó Ay crear una línea dinástica propia en detrimento de Horemheb? En las próximas páginas haremos referencia a algunas de estas incógnitas, pero solamente nos centraremos en los reinados de los 3 últimos reyes de la xvIII dinastía; Tutankhamón, Ay y Horemheb.

6 Estos enigmas están en relación con la figura de Nefertiti. Según HaRRIS $(1974 ; 1992)$ y SAMSON (1978; 1982; 1982a), Nefertiti tuvo un reinado propio tras el año 12 , siendo identificada con el Faraón Neferneferuaten, que aparece junto a Akhenatón en algunos textos y escenas, siendo este, significativamente, el mismo nombre que Nefertiti tuvo desde el año 6 de Akhenatón. En el centro del debate están un grafito tebano de la tumba de Pare (GARDiner 1928); la "estela de la corregencia" (STEWARD 1976: figs. 12; 52) y la estela del soldado Pasi (ALFRED 1968: 244).

En la estela del soldado Pasi, posterior al año 12, aparecen dos faraones bajo los rayos del disco solar, en una escena calificada de homosexual, y donde la figura más grande corresponde a una mujer, Nefertiti según HARRIS y SAMPSON. Según ellos, a partir del año 13 Nefertiti desaparece de las menciones oficiales debido a su nuevo papel como corregente, siendo el título de "Gran esposa real" transferido a Meritaten. Los contrarios a la identificación con Nefertiti señalan que estas escenas nos muestran a las figuras reales desnudas de cintura para arriba, lo que se arguye en contra de la identificación con Nefertiti, pero Ertman (1992) ha demostrado que existen escenas de Nefertiti, anteriores al año 12, en las que está desnuda de cintura para arriba, como en la ventana de las apariciones de la tumba de Ay, o en algunos talatats conservados en Boston, lo que, en opinión de Ertman, confirma que Nefertiti actuó como co-rey durante el período amarniense.

Alfen (1991: 78) niega que este faraón, Neferneferuaten, sea Nefertiti ya que sus hijas siguen refiriéndose a ella como «esposa principal» en tiempos de Tutankhamón y, además, esta identificación implicaría que Nefertiti estaba casada con su propia hija, una perversión excesiva incluso para el período amarniense, como apunta Redford (1984: 192), mientras Murname (1977: 176-8), piensa que Neferneferuaten, sería el primer nombre de Smenkhare, opción que es la más aceptada y plausible, cambiándolo en el año 15 ó 16 de Akhenatón, cuando Akhenatón murió o delegó el gobierno en Smenkhare, ya que la corregencia entre ambos es también objeto de debate. De confirmarse esta posibilidad, implicaría que tras el año 12 la corte amarniense inició los pasos necesarios para restablecer la "normalidad" en el país, ya que en el grafito tebano del "año 3" de la tumba de Pare se menciona a Amón en relación con el templo funerario de Neferneferuaten. Finalmente, objetos con el nombre de Neferneferuaten, presente o alterado, también fueron depositados como ajuar en la tumba de Tutanjamon (Beinlich \& Saleh 1989). 


\section{Tutakhamón}

El corto reinado, 9 años, de este faraón ha pasado a la historia e imaginación popular por el descubrimiento de su tumba y, en segundo lugar, por la petición de su viuda, Ankhesamón, de un marido a Suppiluliuma. Pero la verdadera importancia de su reinado radica en que a través de sus decretos y actividad constructora puede obtenerse una visión, parcial, de la situación en Egipto tras el reinado de Akhenatón. Por otra parte, y al igual que con la mayoría de los personajes de este período, el primer problema con el que nos enfrentamos es su origen y si, como es generalmente aceptado, Smenkhare fue su hermano.

Basándose en análisis realizados a la momia de Tutankhamón y en la adscrita a Smenkhare, se ha defendido que eran hermanos, con un mismo grupo sanguíneo, A2, y similitudes en la estructura ósea (Harrison \& Abdalla 1972; Harrison 1966). Pero Reeves (1990: 42-49) niega que la momia hallada en la tumba KV 55 sea la de Smenkhare, basándose en las dificultades para determinar la edad de las momias (Robins 1981) ${ }^{7}$, defendiendo que la persona allí enterrada fue Akhenatón (Reeves 1982), explicándose el mismo grupo sanguíneo al tratarse de padre e hijo, Akhenatón-Tutankhamón, permaneciendo la tumba de Smenkhare oculta todavía. Sin embargo, en los últimos años parece haberse cierto consenso en que la persona enterrada en esta tumba fue Smenkhare ${ }^{8}$, aunque ello no confirma que fuera hermano de Tutankhamón, aunque si pertenecientes a la misma familia.

\footnotetext{
Una relación familiar podría establecer analizando las distintas momias pero, como es sabido, la mayoría de las momias reales fueron "escondidas" a comienzos de la XXI dinastía, procediéndose a su "restauración", lo que distorsionó muchas de sus características físicas así como su identificación. A pesar de ello, Wente y Harris (1992: 12-14), por ejemplo, creen haber encontrado elementos suficientes para establecer una relación física entre Tutmosis IV y Tutankhamón, aunque estos datos deben ser utilizados con mucha cautela, a pesar de que el culto real más importante en el-Amarna, aparte del de Amenofis III, sea el de Tutmosis IV (Bryan 1991: 161-4).

\& La tumba KV 55 fue iniciada antes del traslado a el-Amarna y sellada durante el reinado de Tutankhamón, siendo originalmente adscrita a la reina madre Tiye (Davis 1910). Las excavaciones de Kemp en la villa de los obreros de el-Amarna (1983-1987) han confirmado que, a pesar del abandono oficial de Akhetaten, posiblemente en el año 2 de Tutankhamón, en la villa de los obreros hubo un nuevo período de ocupación y de actividad constructora, en especial de capillas (Bonnan 1991), por lo que las personas que allí vivian creían que su estancia no iba a ser temporal. Por otra parte, la villa es ahora ocupada por unidades del ejército, posiblemente encargadas de proteger y vigilar el traslado de bienes, documentos, etc., a la nueva capital de Egipto, asi como la necrópolis real y privada. Respecto a estas últimas, la mayoría de las tumbas están inacabadas y suele señalarse que la tumba real no llegó a ser utilizada, aunque MARTIN (1989a) piensa que en ella fue enterrada al menos la reina madre Tiye, permaneciendo la incógnita sobre el lugar de enterramiento de Akhenatón.
} 
Respecto a la paternidad de Smenkhare, la hipótesis más aceptable señala que fue hijo de Amenofis III y Sitamun, su hija y esposa en los últimos años de reinado, lo que adquiere cierta verosimilitud por las siguientes razones:

A) Si Smenkhare tenía 20 años cuando murió y reinó durante 3 años, ello implica que nació el mismo año en el que Akhenatón ascendió al trono de Egipto.

B) Su edad al morir hace difícil pensar que fuera hijo de Kiya, mientras que si fuera hijo de Nefertiti resulta extraño no encontrar representaciones suyas en el-Amarna junto a sus hermanas, a no ser que durante esos años Smenkhare deba ser identificado con Neferneferuaten ${ }^{9}$.

C) El ser hijo de Amenofis III explicaría el que se convirtiera en Faraón de Egipto, ya que su origen le convertía en una persona idónea para iniciar el acercamiento a las antiguas tradiciones. Igualmente, ello explicaría las similitudes físicas entre Smenkhare y Tutankhamón, al proceder de la misma familia - Amenofis III y Akhenatón-, lo que explica su mismo grupo sanguíneo. Además, según Reeves (1992: 47), la enfermedad que Akhenatón tenía estaba ya presente en Tutmosis IV y Amenofis III, así como en Smenkhare y Tutankhamón, enfermedad que explicaría el final de la línea familiar al trono de Egipto, así como el progresivo realismo, o distorsión, del canon artístico egipcio desde Tutmosis IV (Berman 1990).

Por tanto, Smenkhare posiblemente era hijo de Amenofis III y Sitamun, los mismos padres que Varille (1940) y Fairman (1972) defienden para Tutankhamón, pero esta hipótesis descansa en la aceptación de una corregencia entre Amenofis III y Amenofis IV, lo que actualmente no es aceptado ${ }^{10}$.

En cuanto a la paternidad de Tutankhamón, una posibilidad a veces defendida es que Ay fuera su padre, al aparecer en muchas inscripciones

Por otro lado, Smenkhare, fiel a su política de restablecimiento de la normalidad religiosa y política del pais, posiblemente se hizo enterrar en KV 55, reutilizando parte del ajuar originalmente realizado para Kiya (ALLEN 1988), una esposa secundaria de Akhenatón (REEVES 1988), debido a su prematura muerte que impidió la fabricación de un ajuar funerario propio. En los últimos años se ha aceptado que en KV 55 pudo haber dos enterramientos separados en el tiempo, primero al de Tiye (REEVES 1990; BELL 1990) y finalmente el de Smenkhare. De confirmarse su utilización por Tiye, o Kiya, seria el primer enterramiento femenino, no sólo en el valle de los Reyes, sino en la orilla oeste de Tebas, ya que el valle de las Reinas comenzó a ser utilizado en la xlx dinastía.

Finalmente, Dodson (1992a; 1993) ha señalado que Smenkhare murió antes que Akhenatón, lo que explica el ajuar amarniense de la tumba, pero su hipótesis es dudosa.

9 Cf., nota 6.

10 El último intento de ALDRED (1988) por defender dicha corregencia ha tenido escasa aceptación. 
como «padre», pero este título debe entenderse como tutor o instructor (Schaden 1977). La hipótesis más aceptada actualmente es que Tutankhamón era hijo de Akhenatón y Kiya. El que fuera hijo de Akhenatón parece confirmado por las inscripciones de talatats precedentes de Hermópolis donde encontramos la frase "hijo real de su cuerpo" (Cooney 1965). Respecto a Kiya, Martin cree ver en la tumba real de Amarna la representación de su muerte dando luz a Tutankhamón, por lo que Akhenatón tendría por fin un sucesor varón.

La identificación de Kiya es otro de los enigmas de este período, pudiendo ser una princesa de Mitanni enviada por Tusratta a Amenofis IV con el fin de mantener los lazos de amistad con Egipto: Gilukhepa (Redford 1984: 150) o Tadukhepa (Manniche 1975). Su importancia en la corte amarniense es confirmada por el hecho de que parte del ajuar de la tumba KV 55 fue realizado para ella (Eaton-Krauss 1986), como el sarcófago, posteriormente utilizado por Smenkhare (Allen 1988: 21-6; EatonKrauss 1992). Según Reeves (1988), Kiya adquiere importancia en la corte amarniense entre los años 9 y 11 de Akhenatón, por lo que si en verdad fue la madre de Tutankhamón, ello concuerda con su edad cuando ascendió al trono, 9 años ". Una de las dificultades existentes es que entre las escenas de Kiya conservadas, ningún varón es representado (Reeves 1988), si siendo representada con una hija, la cual podría ser Baketatón (Gabolde 1992) ${ }^{12}$. Sin embargo, tampoco se conocen representaciones de Akhenatón o Smenkhare como príncipes o personas cercanas al Faraón, lo que no debe sorprendernos ya que, con anterioridad a los ramesidas los príncipes herederos no suelen ser representados (Dodson 1990). Por tanto, Smenkhare fue posiblemente hijo de Amenofis III y. Sitamun mientras Tutankhamón de Akhenatón y Kiya, respondiendo sus similitudes físicas por pertenecer a la misma línea familiar.

Respecto a su reinado, la primera incógnita es determinar si traslado a Tebas la capital de Egipto o si, por el contrario, esta radicó en Menfis. Los defensores de Menfis (Malek 1985; 1988), señalan que es significativa la importancia que adquiere la necrópolis de Saqqara con posterioridad a Akhenatón (Dijk 1993; Martin 1989), mientras que los defensores de Tebas argumentan las importantes construcciones realizadas en ella por

\footnotetext{
Akhenatón reinó 17 años y Smenkhare 3, teniendo entonces Tutankhamón 9 años. Más problemas plantea su edad a su muerte, señalándose generalmente que murió a los 18 años (FAIRMAN 1972), aunque análisis realizados en 1978 elevan esa edad a entre 23 y 27 (Wente \& Harris 1992: 11), no aceptada por la comunidad egiptológica (EATON-Krauss 1984).

12 A la muerte de Kiya, muchos de sus objetos fueron usurpados o reutilizados por Meritaten, hija de Nefertiti y esposa de Smenkhare.
} 
Tutankhamón, así como el cambio de la partícula Atón por la de Amón en el nombre real en su segundo año de reinado, tras el abandono de elAmarna.

Sin querer adentrarnos en dicha polémica, todo parece indicar que Menfis pasó a ser la capital administrativa de Egipto, no solo porque el famoso Decreto de Restauración de Tutankhamón (CG 34183) se emitiera desde el dominio de Aakheperkare, la fundación de Tutmosis I en Menfis (Dijk \& Eaton-Krauss 1986: 35) ${ }^{13}$, sino también porque tanto Tutankhamón como Horenheb se desplazan a Tebas para la celebración de la Fiesta de Opet, indicando ello que no residían normalmente en la capital religiosa de Egipto ${ }^{14}$. Por otra parte, los altos funcionarios se entierran en Saqqara (Dick 1993), y no en el valle de los nobles de Tebas, lo que confirma a Menfis como centro administrativo de Egipto ${ }^{15}$. El que Menfis fuera la capital de Egipto, no implica un abandono de Tebas y sus templos, ya que como centro religioso, y funerario, de Egipto seguiría disfrutando de un status especial, mientras que el cambio de la partícula Atón y la vuelta a Amón, indica solamente un deseo de volver a la "ortodoxia" ${ }^{16}$.

En relación a su reinado, se observa una ruptura política y administrativa con la tradición Amarniense, al mismo tiempo que una continuidad en algunos aspectos. La principal fuente de información sobre su gobierno la constituye la Estela de restauración (Legrain 1907), donde repone a la antigua nobleza en sus cargos, restituyéndoles sus propiedades y status, reabre los templos cerrados durante la «herejía» y nombra para los altos cargos sacerdotales a hijos de "personas conocidas" ${ }^{17}$. Aparte del posible

13 A pesar de que dicha estela fue hallada en Karnak.

${ }_{14}$ En la XIX dinastía los ramesidas establecerán definitivamente su capital en el Norte y se desplazarán periódicamente a Tebas para cumplir con sus obligaciones religiosas o funerarias. Por otra parte, la consideración de Tebas como capital religiosa y del Bajo Egipto como centro político y administrativo puede remontarse a la XII dinastía.

15 El propio Horemheb, antes de acceder al trono de Egipto, construyó su tumba en Saqqara (MARTIN 1989). El abordar las consecuencias y razones para el abandono del Valle de los Nobles en Tebas, así como el hecho de que por primera vez en Egipto, aparte de los periodos de descentralización, se separen geográficamente los enterramientos de nobles y reyes, nos apartaría de nuestra temática, pudiéndose consultar al respecto el trabajo de Dijk (1988) y, especialmente, el magnífico libro de Assman (1984), innovador en muchas de sus concepciones.

${ }_{16}$ Por otra parte, los defensores de Tebas como capital de Egipto olvidan que Tutankhamón fue el único Faraón que volvió a utilizar la parícula Amón, al mismo tiempo que ya desde su reinado se observa una tendencia a favorecer y proteger a otras divinidades, como Ptah, que se encuentran más cercanas a ia realeza, posiblemente por el hecho de ser Menfis la capital de Egipto.

27 Suele señalarse que Akhenatón se rodeó de funcionarios medios o bajos, lo que explicaría en parte el fracaso de sus reformas. Sin embargo, no hemos de olvidar que, al igual que sucedió con el reinado de Hatshepsut y el posterior de Tutmosis III, ningún noble o funcionario 
contenido propagandístico de algunas de las medidas y frases contenidas en estos decretos, todo apunța a que la situación en Egipto era mala administrativamente, ya que estas mismas medidas son repetidas por Horemheb en su famoso Decreto (Kruchten 1981) y en cierta medida por Seti I (Leprohon 1985).

Sin embargo, no hemos de olvidar el trasfondo propagandístico de estas declaraciones públicas y oficiales. Así, será característico de los faraones de la XIX dinastía señalar que sus acciones de gobierno van encaminadas a reforzar el poder de Egipto y el funcionamiento de la administración, como si hubieran existido problemas en los reinados precedentes, de igual forma que todos los reyes del Imperio Nuevo declaran haber extendido las fronteras de Egipto aun cuando algunos de ellos no llegarán a realizar campaña militar alguna ${ }^{18}$.

La actividad constructora y económica en los templos es retomada, no sólo en Tebas, sino en todo Egipto, como prueba el decreto emitido en favor de Maya en su año 8 de reinado, por el que este alto funcionario es encargado de establecer ofrendas en todos los templos, desde Aswan hasta el Mediterráneo (Amer 1985), la misma medida que adoptara Horemheb. Igualmente, inicia la restauración de los nombres borrados durante el reinado de Akhenatón (Barguet 1962), acción atribuida normalmente a Horemheb, pero que usurpó el nombre de Tutankhamón en esta labor, así como en la propia Estela de Restauración (Gabolde 1987) y en otros monumentos como veremos.

De la política constructora de Tutankhamón se desprende un deseo por terminar las construcciones iniciadas por Amenofis III, como la columnata del templo de Luxor y el templo de Soleb en Nubia, quizá en un intento por relacionar su persona y reinado con Amenofis III olvidando el interregno amarniense. En este contexto se extiende, por ejemplo, que termine la decoración del III pilono de Karnak, representándose en él como Faraón junto a Amenofis III o, más significativamente, que construya templos funerarios

\footnotetext{
haría referencia a su posible intervención en la herejía amarniense. Por otra parte, los textos en los que los funcionarios dicen haber sido elevados a los altos cargos de la administración copian y responden a modelos del Imperio Antiguo e, incluso, como el propio Horemheb en su tumba menfita, construida y decorada cuando ya había sido reestablecida la "normalidad" en el país.

${ }_{18}$ En cualquier caso, el texto de esta Estela constituye una ruptura con toda la tradición egipcia anterior en lo referente a juzgar el gobierno de los predecesores, siendo esta una de las numerosas consecuencias del período amarniense. Así, por ejemplo, tras los acontecimientos del Primer Período Intermedio lo único que se le dice al rey es que reestablezca el orden, no que la carencia de orden sea una consecuencia de su gobierno; cf., Griffiths 1991: 168-73.
} 
para el culto a Amenofis III en Faras y Kawa, Nubia ${ }^{19}$. Pero no se limitó a terminar lo ya iniciado (Eaton-Krauss 1988), siendo suya la idea de unir el $X$ pilono de Karnak con el templo de Mut mediante una avenida de esfinges (Berlandini 1980), además de realizar obras en este mismo pilono (Redford 1983).

Pero, sin lugar a dudas la construcción más importante de Tutankhamón fue el recinto llamado «kwt Nb-hprw-R'm Wçst», "Mansión de Nebkheperure en Tebas", proyecto continuado, no usurpado como piensa Redford (1984: 206), por Ay. De este recinto, posteriormente desmantelado por Horemheb, se conservan escenas muy interesantes, como las referidas a Nubios y Asiáticos, algo que Redford (1983) toma como prueba de actividad militar exterior tras el reinado de Akhenatón. Igualmente, Eaton-Krauss (1988) incide en la importancia de una escena en la que Tutankhamón está ofreciendo Maat al modo y manera de como será característico en época ramesida, indicando ello la adopción de nuevas escenas e iconografías que perdurarán en el tiempo y caracterizarán la posterior historia de Egipto ${ }^{20}$.

Un aspecto importante es la posibilidad de que Tutankhamón iniciará la utilización de. las construcciones de Akhenatón en Tebas, sin que ello implique una persecución de los posibles recuerdos de su reinado, ya que la utilización de bloques antiguos en nuevas construcciones es una costumbre normal en el Imperio Nuevo e incluso antes (Weinstein 1973). Dicha posibilidad parece confirmarse tras los estudios realizados en los restos de la avenida de esfinges erigida entre el $X$ pilono y el templo de Mut en Karnak, esfinges que originalmente fueron realizadas por Akhenatón (Eaton-Krauss \& Murname 1991) ${ }^{21}$.

Pero, el aspecto que más nos interesa en relación a la sucesión real es el papel que en el reinado de Tutankhamón tuvieron Ay y Horemheb.

19 Este aspecto es de gran importancia ya que con ello se inicia una de las actitudes señaladas para los Faraones del Imperio Nuevo respecto a Nubia, y no seguida, por ejemplo, en el propio Egipto o en Siria-Palestina: la identificación del Faraón, proceso que alcanzará su punto álgido con Rameses II (HABACHI 1969).

20 Este tipo de escenas, significativamente, son muy comunes en los años de tebanos de Akhenatón, como demuestran las escenas conservadas del Festival Sed (GoHARY 1989), desapareciendo cuando traslada la capital a el-Amarna.

${ }_{21}$ Originalmente, estas esfinges tenian cabezas femeninas, alas tripartitas y la musculatura del cuerpo es masculina, por lo que podrian representar a Nefertiti y Akhenatón. Este descubrimiento, no viene sino a confirmar el importante papel que desempeñó Nefertiti en el período amarniense, algo que ya era conocido por las representaciones de Talatats (REDFORD 1977), así como por las escenas del Festival Sed celebrado por Akhenatón en Tebas en su tercer año de reinado (GOHARY 1992). 
1) Ay tiene ya gran importancia con Akhenatón, encontrándose en su tumba de el-Amarna el famoso himno a Atón (Davies 1908). Tiende a considerarse que Ay fue sacerdote, cuando en realidad desarrolló la carrera militar (Schaden 1977), teniendo el título de "padre del Dios" ya con Akhenatón, lo que se intentó relacionar con la posibilidad de que Ay fuera el padre de Nefertiti, ya que su esposa, Ty, tiene el título de «niñera de la Gran Esposa del Rey, Neferneferuaton, Nefertiti». Pero el título de «padre del Dios" debe entenderse como tutor, instructor del rey ${ }^{22}$. Sorprendentemente, Ay desaparece de la documentación amarniense en el año 9, permaneciendo su tumba inacabada ${ }^{23}$ y apareciendo públicamente de nuevo con Tutankhamón.

Seele (1955), intentó explicar esa ausencia de Ay en la última mitad del reinado de Akhenatón aludiendo a la posibilidad de que se encontrara fuera de Egipto por motivos de estado, ya que en algunas cartas del archivo diplomático de el-Amarna encontramos referencias a un oficial llamado ha, ha hai, haia haya, equiparado por Knudtson (1908-15: 1030) con Ay. Sin embargo, estas cartas (EA 11; 66; 71; 101; 109; 112; 117; $166-7 ; 255$; $268-9)$ pertenecen a la primera mitad del reinado de Akhenatón (Campbell 1964) y posiblemente hagan referencia a Huya, aparte de que su posible ausencia de Egipto no explica la paralización de las obras en su tumba. Es por ello por lo que podemos plantear que Ay, tras el año 9, cayó en desgracia en la corte Amarniense, posiblemente por presentar reticencias al giro radical que adoptó la ideología de Akhenatón tras ese año, algo que, posiblemente, le permitió volver a participar en la política egipcia tras la muerte de Akhenatón.

Con Tutankhamón, a los títulos que ya tenía añade el de "líder del Festival de la Eneada, padre del Dios Ay», cargo que no es sacerdotal sino otorgado a alguien temporalmente para la realización de un Festival (Schaden 1977: 138), conservando el título de "padre del Dios", en referencia a su papel como instructor del rey. Pero, la importancia de Ay con Tutankhamón la encontramos reflejada en una escena en la que Tutankhamón, seguido por su esposa Ankhesamón, está derrotando a un enemigo, estando Ay representado delante del rey, en una posición reservada siempre para la divinidad (figura 1). Sin embargo, y a pesar de que pudiera pensarse lo contrario, Ay no desempañó el cargo de visir con

z2 Sobre este título, sus connotaciones y atribuciones en períodos anteriores; cf., Habachi (1958), Blumenthal (1987).

${ }_{23}$ Como lo confirma el que en su tumba de el-Amarna sólo encontramos las referencias a Atón bajo su fórmula primitiva, y no la oficial con posterioridad al año 9 . 


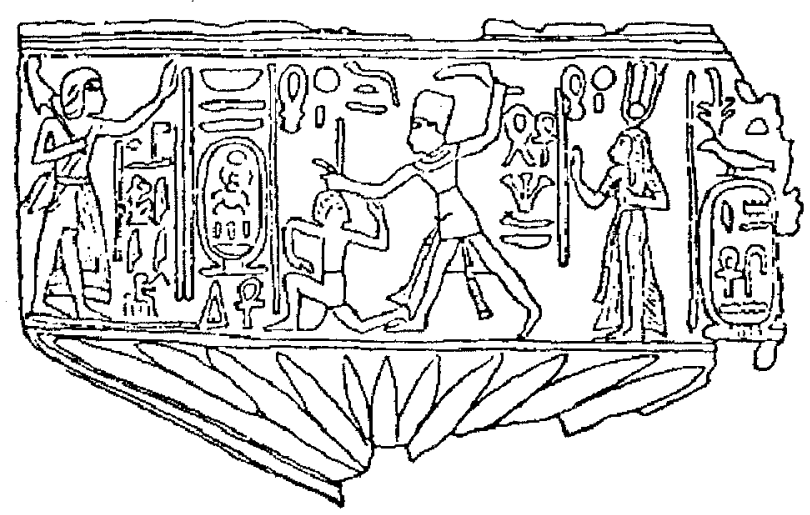

Figura 1. Tuntankhamon venciendo a sus enemigos en presencia de Ay. Tablilla procedente de KV 58 (JE 57438).

Tutankhamón, ya que estos fueron Penthu y Usermont (Habachi 1979), aparte de Huy que fue virrey de Kush.

2) Respecto a Horemheb, ha habido algunos intentos por identificarle con Paatenemheb, un funcionario de el-Amarna y Schulman (1979) piensa que pudo participar en la campaña nubia de Akhenatón contra el país de Ikayta realizada en el año 12 (Smith 1976).

Las fuentes sobre Horemheb antes de llegar a ser Faraón son escasas, destacando su tumba menfita (Martin 1989) ${ }^{24} \mathrm{y}$, especialmente, su texto de coronación (Gardiner 1953), donde leemos que durante años gobernó Egipto por encargo del rey, teniendo el título de «príncipe heredero» e «hijo mayor de Horus», es decir, teóricamente era la persona destinada a suceder a Tutankhamón. Puede arguirse que estos títulos son propagandísticos, pero también los encontramos en su tumba menfita, finalizada antes de llegar a ser Faraón ${ }^{25}$, y donde en una escena es recompensado por Tutankhamón (figura 2). Lógicamente, también debemos pensar que, en teoria, Horemheb disfrutaba de esa consideración hasta que Tutankhamón tuviera un sucesor directo ${ }^{26}$.

${ }_{24}$ Sobre la figura de Horemheb como príncipe existen dos monografías, Pflüger (1936) y Hari (1964), ambas desfasadas por no haber dispuesto de la documentación de su tumba y solamente de bloques aislados procedentes de la misma. La tumba de Horemheb fue redescubierta en 1975.

25. Para una discusión de los títulos y su veracidad histórica, cf., Dijk, 1993: 11-24.

${ }_{26}$ Con Tutankhamón finalizó la línea dinástica que podía remontarse a Kamose y reyes de la XVII dinastía. En la tumba de Tutankhamón se encontraron dos sarcófagos conteniendo fetos de pocos meses. 


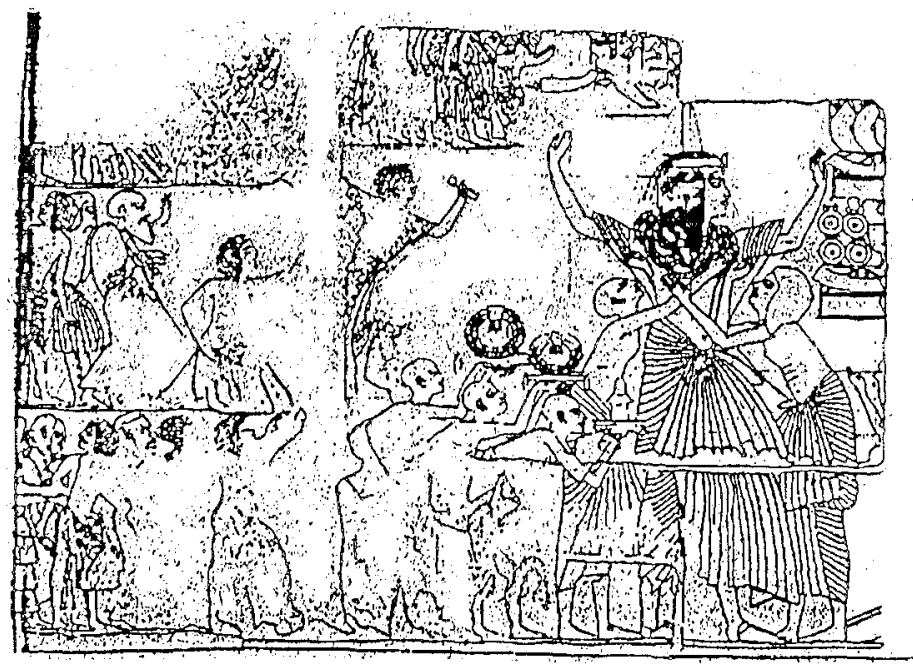

Figura 2. Relieve de la tumba menfita de Horemheb siendo recompensado por Tutankhamón. Martin 1989, fig. 47.

Por tanto, encontramos que tanto Ay como Horemheb desempeñaron importantes funciones durante el reinado de Tutankhamón, teniendo más poder Horemheb, por lo que el ascenso de Ay al trono puede considerarse, en cierto sentido, un golpe de Estado.

La muerte de Tutankhamón ha sido analizada casi exclusivamente desde la perspectiva del conocido episodio de su viuda, Ankhesamón, pidiendo a Suppiluliuma un marido. Recientemente, Reeves (1990: 33) ha sugerido que Tutankhamón pudo morir violentamente por los deseos de Ay de convertirse en Faraón, pero las causas de su muerte siguen siendo un misterio (Eaton-Krauss 1990: 554-5).

La hipótesis que más aceptación tiene es la de que a la muerte de Tutankhamón Horemheb se encontraba fuera de Egipto, lo que fue aprovechado por Ay para ascender al trono (Helck 1981; Welsh 1993). El debate está centrado en dos hechos, cuyo análisis nos apartaría en exceso de nuestra temática; la identidad de la reina que pide un marido a Suppiluliuma ${ }^{27}$ y los posibles choques armados entre Egipto y Hatti a la

${ }^{27}$ En los últimos años se ha reabierto la polémica sobre la identidad del rey de Egipto mencionado en las "Lamentaciones de Suppiluliuma" en las que se recoge su muerte (Güterbock 
muerte de Akhenatón, aspectos que todavía necesitan nuevas investigaciones y que no vienen sino a complicar aún más este período de la historia de Egipto con nuevos interrogantes ${ }^{28}$.

Un último aspecto a analizar, de forma somera, es el de la tumba de Tutankhamón, KV 62, la más pequeña de todo el valle de los Reyes, y la información histórica que de ella puede obtenerse ${ }^{29}$.

Ya hemos señalado los intentos de Tutankhamón por relacionar su persona y reinado con Amenofis III, algo que impidió la prematura muerte del Faraón, quién se vio obligado a descansar en una tumba más modesta, posiblemente una de las tumbas inacabadas que en un momento determinado se comenzaban y, que por distintas razones no se terminaban o permane-

1956). Como es sabido, la identidad de este rey suele adscribirse a Tutankhamón pero, en los últimos años, la escuela alemana, encabezada por Krauss (1978: 9-19) y seguida por Sürenhagen (1985: 41) y Meyer (1993), ha puesto de relieve la posibilidad de que esta petición de matrimonio no fuera realizada por la viuda de Tutankhamón ya que el rey egipcio que en las fuentes Hititas es mencionado como muerto debe ser identificado con Akhenatón, mientras que Wilhelm \& Boese (1987) apuntan la posibilidad de que la petición corresponda al reinado de Smenjare, y Krauss (1978: 33-47) apunta a Meritaten como la príncesa que envió la carta a Suppiluliuma.

En las fuentes hititas, el rey egipcio es llamado Nipkhururia, que los defensores de que en realidad se trata de Akhenatón señalan es una transcripción al acadio de Neferkheprure (Akhenatón). Sin embargo, Bryce (1990) ha vuelto a defender que es Tutankhamón el rey muerto, mientras Murname (1990: 115-37), tras analizar todas las fuentes y establecer la cronología de los acontecimientos, no se decanta en un sentido u otro a la espera de mayor información.

28 Entre esos nuevos interrogantes podemos señalar:

A) Si en verdad existió un enfrentamiento entre Egipto y Hatti en el reinado de Tutankhamón, ¿cómo se explica que las vías de comunicación entre ambos reinos sean tan permeables y que, en medio de luchas por el control del Norte de Siria, Egipto ofrezca el trono a un asiático? Máxime si, como señalan los defensores de la opción de Tutankhamón, poco antes de su muerte Egipto había sufrido una derrota en Amqa.

B) Si la petición de un marido parte de la ciuda de Tutankhamón, ¿no podría ello ponerse en relación con el hecho de que Tutankhamón era hijo de una princesa asiática, Kiya, lo cual influiría en su concepción de la realeza y chocaría frontalmente con los ideales egipcios de la misma?

C) Si en verdad Ankhesamón pidió un marido a Suppilulima, ¿cuál sería la razón de Horemheb, teóricamente el sucesor de Tutankhamón por su titulatura?

D) ¿En qué época del año se produjo la muerte de Tutankhamón y su posterior enterramiento?, ¿se respetaron los 70 días que duraban los rituales funerarios o, por el contrario, Ay los aceleró para asumir el trono de Egipto aprovechando que Horemheb estaba fuera de Egipto?

E) Si, como los análisis botánicos realizados en la tumba y ajuar de Tutankhamón revelan (GERMER 1989; HePper 1990), su muerte se produjo a finales del verano y el enterramiento a finales de la primavera siguiente, ¿por qué no volvió inmediatamente Horemheb para ocupar el trono?

F) ¿Por qué Ay no llegó a casarse nunca con Ankhesamón? Es cierto que existen objetos en los que los dos aparecen juntos, pero su mujer Ty sigue siendo mencionada en los textos como Esposa del Faraón.

${ }^{29}$ Esta no fue la única tumba de Tutankhamón, iniciándose la construcción de su tumba en elAmarna antes de su traslado a Menfis, pudiendo ser la misma la tumba 27, cf, el Khouli \& Martin, 1987: 15. 
cieron sin decoraciones o textos (Ryan 1992). Respecto el ajuar funerario de Tutankhamón, lejos de aclararlos algunos aspectos controvertidos de su figura y origen, no hace más que aportar elementos de apoyo a las distintas teorias existentes al tener diferentes procedencias (Reeves 1990; Vandersleyen 1992: 78).

En relación a los acontecimientos políticos anteriormente descritos, debemos recordar que en la tumba de Tutankhamón encontramos ushebtis dedicados a su culto funerario depositados por altos funcionarios como Maya o Nahktmin, pero ninguno de Horemheb, bien porque este se encontrara fuera de Egipto en el momento de su muerte o porque Ay le relegó.

Ay

Como hemos ido señalando, su figura y personalidad está rodeada de un velo enigmático. En su tumba de Amarna encontramos cuatro títulos: "Verdadero escriba real", «inspector de caballos", y "padre del Dios" (Schaden 1977), todos ellos relacionados con el ejército, algo puesto en relación por algunos con las reformas en el ejército que realizó Tutmosis IV y la creación de un cuerpo diferenciado de carros de combate (Bryan 1991; Schaden 1977), mientras que el cargo de "padre del Dios" ya ha sido analizado. Su origen, y el de su esposa Ty, es oscuro, pero probablemente proceden del Egipto medio, concretamente de la región de Akhmin, donde Ay erigió una estela como rey.

Respecto a su labor de gobierno y política de restablecimiento del culto a Amón poco sabemos; realizó construcciones destinadas a su culto y completó otras iniciadas por Tutankhamón, como la «Mansión de Nebkheperure en Tebas» (Schaden 1977). Nuestro desconocimiento procede, en parte, de la destrucción, o reutilización, de sus monumentos por parte de Horemheb, encontrando en diversos monumentos el nombre de Ay intencionadamente borrado.

Esta posible "damnaatio memoriae» contra Ay llegó a alcanzar su tumba, aunque en opinión de Reeves (1990: 71-2), dicha persecución fue realizada en época ramesida. Sin embargo, Dodson (1992: 55-6) ha señalado, acertadamente en nuestra opinión, que dicha persecución debió realizarse más cercana en el tiempo a $\mathrm{Ay}$, ya que en época ramesida no habría memoria de los acontecimientos ocurridos y, si la persecución hubiera ido encaminada contra los faraones de Amarna, ¿por qué tumbas como la de Tutankhamón u otras permanecieron inalteradas? Es cierto que en sus inscripciones Horemheb dice haber terminado la tumba de 
Ay, pero estas declaraciones son normales si consideramos que Horemheb no tenía ascendencia real y debía aparecer como legítimo sucesor al poder de Egipto, por lo que la suposición de Dodson (1992: 55-6) de que dicha persecución fue realizada por Seti I o Rameses I parece descartable.

La posible respuesta a esta "damnaatio memoriae» puede estar, no sólo en su subida al trono tras la muerte de Tutankhamón, sino también en su intento de crear una línea dinástica propia, en detrimento de Horemheb, explicando ello la política de este último y su presentación ante la sociedad como el verdadero restaurador del orden divino, llegando a usurpar monumentos del propio Tutankhamón, como la misma estela de restauración.

En el centro de la polémica se encuentra la figura de Nakhtmin ${ }^{30}$, de quien Schulman (1964) señala que fue Vicerrey de Kush con Ay, rebelándose contra él para ser derrotado por Horemheb. Sin embargo, Helck (1981) prefiere otra interpretación; Ay elevó a Nahktmin a un status importante en detrimento de Horemheb, provocando ello la aparición de importantes tensiones.

Recientemente, Ockinga (1991), ha reabierto la polémica tras las excavaciones realizadas en la tumba del noble Sennedjem en Awlad Azzaz, donde se aprecia una persecución de su memoria, perviviendo su nombre solamente en dos lugares inaccesibles de la tumba, mientras que la figura de Tutankhamón no sufrió daño alguno. En una de las escenas conservadas, encontramos la característica escena de la ventana de las Apariciones, debajo de la cual están Sennedjem y su esposa, y en otra un desfile militar en el que Tutankhamón conduce un carro acompañado por otra persona, posiblemente Ay (figura 3 ) ${ }^{31}$. La razón para que esta tumba fue «destruida» puede estar en que por los títulos conservados en ella, así como en los escasos monumentos de Nahktmin que nos han llegado, podemos deducir que Sennedjem era el padre de Nahktmin, afectándole por tanto la persecución de que fue objeto su hijo. Lógicamente, surge una pregunta, ¿si la figura que acompaña a Tutankhamón en el carro es

30 Algunos se refieren a él como "Minnakhte». Como ya hemos señalado, en la tumba de Tutankhamón se encontraron cinco ushebtis suyos como ofrendas, cf., H. BEINLICH \& M. SALEH (1989: 318a, 318c, 330i, 330j y 330k).

${ }^{31}$ Otra posibilidad es que Sennedjem acompañara a Tutankhamón, pero el está representado justo enfrente del carro. También es de destacar que en los textos del Imperio Nuevo encontramos referencias a que el Faraón va en el carro junto a otras personas, en especial en las batallas, pero esta es la primera representación conocida al respecto, anticipando algo que será normal en época ramesida, como confirma que Rameses II diga el nombre de su conductor cuando se encontró solo ante el ejército hitita en Kadesh. 
Ay, por qué no sufrió daño alguno? La respuesta puede estar no en un "olvido" como apunta Ockinga (1991), sino en que esta figura no tenía inscripciones, por lo que no podía ser identificada. Finalmente, debemos recordar que esta tumba está en la región de Akhmin, de donde procedía la familia de Ay.

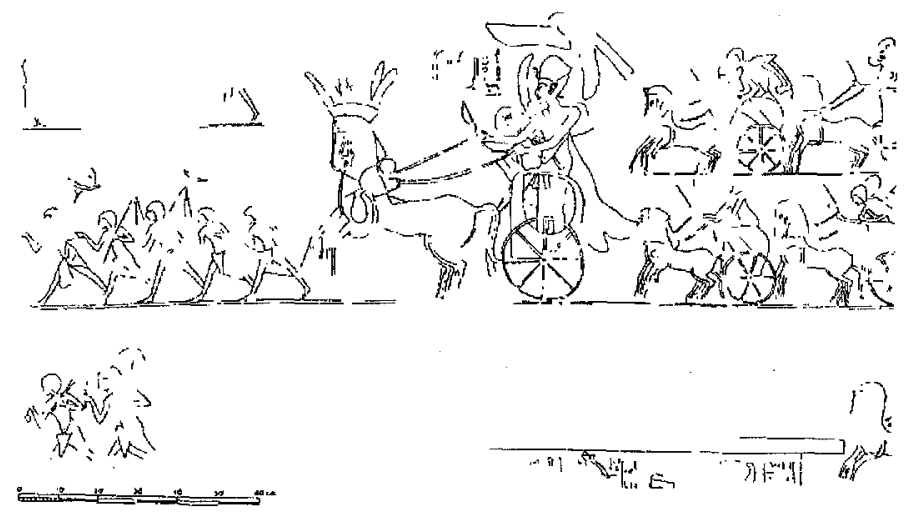

Figura 3. Escena procedente de la tumba de Sennedjem en Awlad Azzaz. Ockinga 1991, fig. 1.

En este mismo contexto, se encuentra una estela privada hallada en Guizah, en las cercanias de la Esfinge, (Dijk \& Eaton-Krauss 1986), con los cartuchos y figuras destruidas, y que está dirigida a Tutankhamón (figura 4). Los candidatos a estar representados en ella son Ay, Horemheb, Maya y Nakhtmin (Dijk \& Eaion-Krauss 1986). Respecto a Ay, en el área menfita no conocemos ninguna representación suya junto a Tutankhamón, al contrario que en Tebas, por lo que no es probable que se trate de él. En cuanto a Horemheb, su figura pudo ser borrada por Ay, pero entonces no se explica por qué su tumba menfita no sufrió daño alguno, quedando Maya y Nakhtmin. El primero disfrutó de una alta consideración real como demuestra su tumba menfita y los títulos adquiridos, por lo que la persecución de su nombre y figura no tendría sentido, quedando solamente Nahktmin, pudiendo disponer en esta estela de otra prueba de la existencia de tensiones entre Ay y Horemheb.

Por lo tanto, el reinado de Ay se caracterizó por un intento de asociar su reinado al de Tutankhamón, presentándose como legítimo sucesor, al mismo tiempo que por sus intentos de crear una nueva familia dinástica, no hemos de olvidar que Tutankhamón era el único descendiente real de la realeza ahmosida. Pero, al igual que en otros aspectos de este período, 


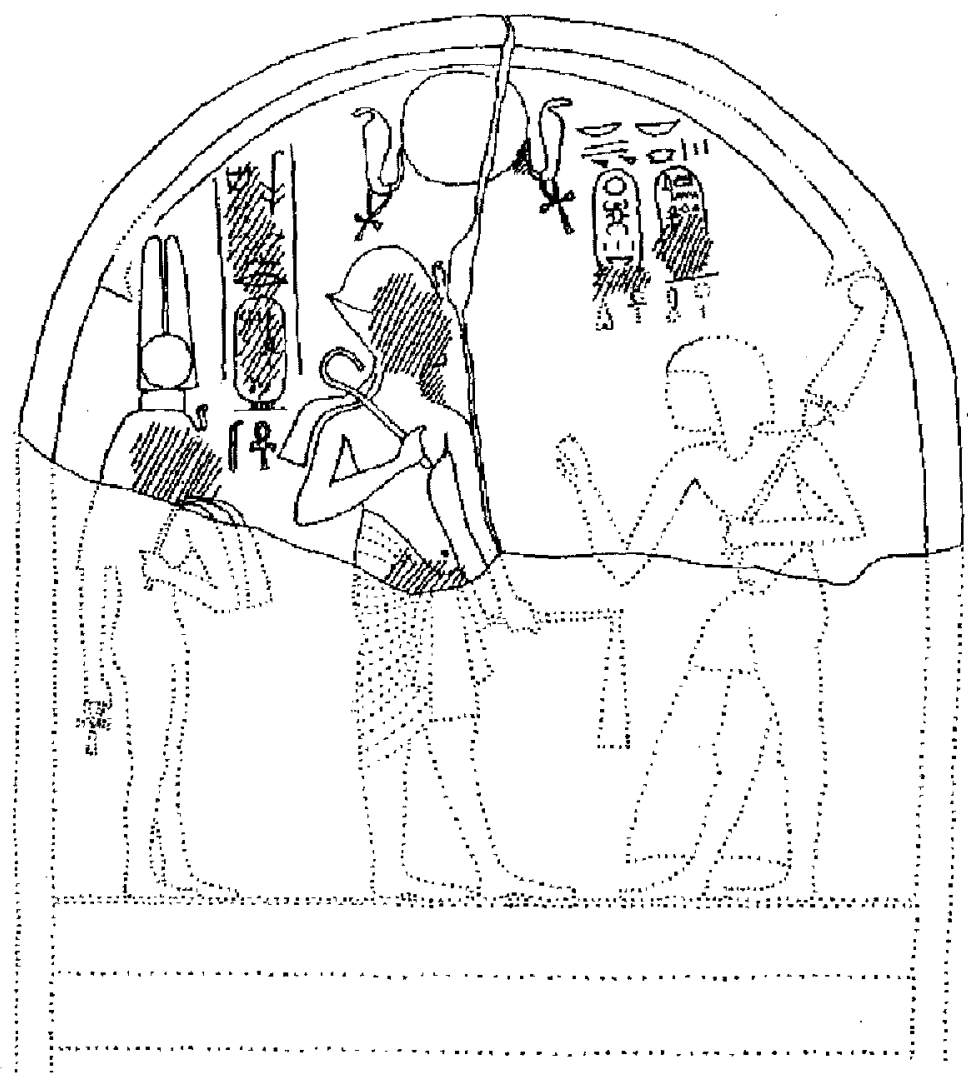

Figura 4. Fragmento de la estela hallada en las proximidades de la Esfinge Eaton-Krauss y Dijk van 1986, fig. 1.

permanecen enigmas por resolver: ¿cómo logró Horemheb hacerse con el trono, cuando, lógicamenie, su figura no disfrutaría de importantes apoyos en la corte de Ay? ${ }^{32}$. Al respecto, Martin (1989: 48) al referirse a las escenas militares de la tumba menfita de Horemheb y, concretamente, aquellas de delegaciones extranjeras presentando su homenaje y tributo, piensa que pueden hacer referencia a las ceremonias relacionadas con el ascenso de Ay al trono, lo que no tiene mucho sentido si en verdad Horemheb fue desplazado del trono por Ay.

32 Al respecto, hemos de señalar que su tumba menfita estaría prácticamente finalizada a la muerte de Tutankhamón. 
La respuesta de que Ay y Horemheb se sucedieron sin tensiones parece descartable, pudiendo encontrar la misma en el apoyo que Horemheb pudo encontrar en el ejército. Al respecto, no hemos de olvidar que en las reformas que Horemheb llevó a cabo durante su reinado, recogidas en su decreto, está la participación de miembros del ejército en los tribunales de justicia, algo nuevo en la sociedad egipcia, al mismo tiempo que Rameses I, futuro rey de Egipto, es representado en su tumba y fue un oficial importante del ejército, sin poder olvidar igualmente el hecho de que en la XIX dinastía, posiblemente hasta la batalla de Kadesh (Assman 1983-4), el ejército tuvo un papel dirigente en el gobierno de Egipto, por encima del todopoderoso clero de Amón que, a pesar de lo que tradicionalmente se piensa, no llegó a disfrutar del poder e influencia que tuvo en la XVIII dinastía.

\section{Horemheb}

Su reinado suele considerarse como aquel en el que la normalidad vuelve a imperar en el Estado egipcio; los cultos y templos de los dioses están plenamente reestablecidos, las acciones militares en el exterior son retomadas, la administración interna vuelve a funcionar, etc., pero en realidad debe considerarse una continuación de lo ya iniciado por Tutankhamón.

Los orígenes de Horemheb son oscuros, sin poder determinar con exactitud si desempeñó algún papel durante el período amarniense como sostiene Schulman (1979) o si puede ser identificado por Paatenemheb, un noble de el-Amarna, debido a las similitudes en sus títulos y nombres; "Aten esta en el Festival", "Horus esta en el Festival».

Respecto a su política, ya hemos señalado que muchos de sus pretendidos actos de gobierno en realidad responden a usurpaciones de estelas pero, lo que si puede establecerse con seguridad, es que con él la política de desmantelación de las construcciones erigidas por Akhenatón en Tebas fue muy importante, a pesar de la opinión de Hari (1985) de que la persecución contra la memoria y monumentos de Akhenatón no comenzó hasta Rameses ${ }^{3}{ }^{33}$.

33 No debemos olvidar que Tutankhamón ya utilizó obras amarnienses para realizar o completar su labor constructora. Por otra parte, debemos diferenciar entre dammatio memoriae y una 
La conclusión que puede extraerse tras todo lo expuesto es que el final del período amarniense, lejos de estar bien conocido, es uno de los periodos que más incógnitas presenta. Es por ello por lo que los trabajos de la Egypt Exploration Society en Menfis y Saqqara, así como los de Kemp en el-Amarna deben proporcionarnos nuevos datos sobre el mismo. No hemos de olvidar que, la historia de Egipto, al igual que en otros períodos, se ha escrito bajo la óptica de una documentación incompleta, procedente en su mayoría de la capital religiosa de Egipto, Tebas, y no de la procedente de los centros administrativos, al mismo tiempo que estas reconstrucciones fueron emitidas en el primer tercio de nuestro siglo, aceptándose durante años y buscando, además, confirmaciones epigráficas en textos cortos, fragmentarios y muchos de ellos propagandísticos. Igualmente, durante décadas la egiptología ha cometido el error de estudiar y explicar lo acaecido en Egipto desde la información egipcia, dejando a un lado la que proporcionaban otros estados próximos orientales que, si bien es cierto, proporcionan una visión radicalmente diferente en ocasiones, pueden servir de contrapunto e inicio de un debate que ilumine lo que realmente sucedió. Como es lógico suponer, muchas de las hipótesis y planteamientos expresados en este artículo pueden ser modificados, confirmados o matizados en los próximos años pero, lo que no cambiará, es la necesidad de reescribir nuevamente la historia de la «revolución amarniense», uno de los períodos de la civilización egipcia que más huella, e imaginación, ha dejado en la historia.

\section{BIBLIOGRAFIA}

ALDRED, C. (1968), Akhenatón Pharaoh of Egypt. A new Study, Londres.

- (1988), Akhenaten. King of Egypt, Londres.

ALLEN, J. (1988), "Two altered Inscriptions of the Late Amarna Period", JARCE 25, 117-26.

- (1989), "The Natural Philosophy of Akhenatón», Religion and Philosophy in Ancient Egypt, Yale Egyptological Studies 2, 89-101.

- (1991), "Akhenaten's mystery coregent and succesor», Amarna Letters 1, 74-85.

AMER, A. (1985), «Tutankhamun's Decree for the Chief Treasurer Maya", RdE 36, 17-20.

Assmann, J. (1983), Re und Amun, Freiburg.

- (1984), Aegypten: Theologie und Frömmigkeit einer frühen Hochkultur, Stuttgart.

- (1983-84), "Krieg und Frieden im alten Ägypten: Ramses II und die Schlocht ber Kadesch», Mannheimer Forum, 175-231.

utilización de materiales, por proximidad, necesidad y facilidad, en la construcción de nuevos templos, pilonos, etc., ya que, por ejemplo, Rameses II no sólo utilizó monumentos de Akhenatón, sino de todos aquellos reyes que habian erigido alguna construcción cercana a las suyas. Al respecto, puede consultarse el sugerente estudio de Malek (1992) en el sentido de que el príncipe Khaemwere, tradicionalmente considerado el primer arqueólogo de la historia, en realidad pudo ser el encargado de obtener los materiales que su padre demandaba para sus construcciones. 
Berman, L. (1990), The Art of Amenhotep III: Art Historical Analysis, Cleveland Museum of Art, 1990.

BARguet, P. (1962), Le temple d'Amon-Re à Karnak. Essai d'exégese, RAPH 21, El Cairo.

BEINLICH, H. \& SALEH. m. (1989), Corpus der hieroglyphischen Inschriften aus dem Grab des Tutanchamun, Oxford.

BEIL, M. (1990), "An Armchair Excavation of KV 55", JARCE 27, 97-137.

BERLANDINI, J. (1980), “Un dromos de Toutânkhamon au X pylône de Karnak», Karnak VI, 19731977,247 ss.

BlumEnTHAL, E. (1987) «Die Gottesväter des Altem und Mittleren Reiches», ZAS 114, 10-35.

Bomann, A. (1991), The Private Chapel in Ancient Egypt, Londres.

BRYAN, B. (1991), The Reign of Thutmosis IV, Baltimore.

Bryce, T. (1990), "The Death of Niphururiya and its Aftermath», JEA 76, 97-105.

CAMPBELL, E. (1964), The Chronology of the Amarna Letters, Baltimore.

COONEY, J. (1965), Amarna Reliefs from Hermopolis in American Collections, Brooklyn.

DAVIES, G. (1908), The rock tombs of El Amarna, VI, Londres.

- (1910), The Tomb of Queen Tiyi, Londres.

DIJK, J. van (1988), "The Development of the Memphite Necropolis in the Post-Amarna period", Memphis et ses nécropoles au Nouvel Empire. Nouvelles données, nouvelles questions, Zivie, A. (Ed.), París, 37-46.

- (1993), The New Kingdom Necropolis of Memphis. Historical and Iconographical Studies, Groningen.

DiJK, J. van \& EATON-Krauss, M. (1986), "Tutankhamun at Memphis», MDAIK 42, 35-41.

Dodson, A. (1990), "Crown Prince Djhutmose and the Royal Sons of the Eighteenth Dynasty» JEA 76, 87-96.

- (1992), "Death after Death in the Valley of the Kings", Death and Taxes in the Ancient Near East, Nueva York, 53-60.

- (1992a), "KV 55 and the End of the Reign of Akhenaten", Atti VI Congresso Internazionales di Egyptologia, Turín 135-140.

- (1993), "On the Origin, Contents and Fate of Biban el-Moluk Tomb 55», GM 132, 21-28.

EAton-Krauss, M. (1983), "Der Bildhauer Thutmose in Amarna", Jahrbuch Preussischer Kulturbesitz 20, 119-13?.

- (1986), «Kija. Ursprüngliche Besitzerin der Kanopen aus KV 55», MDAIK 42.

- (1988), "Tutankhamun at Karnak», MDAIK 44, 1-11.

- (1990), "Akhenaten versus Akhenaten", BiOr 47, 541-559.

- (1992), "The Sarcophagus in the Tomb of Tut'ankhamun», After Tut'ankhamun, C. Reeves (Ed.), Londres, 85-90.

Eaton-Krauss, M. \& Murname, W. (1991), "Tutankhamun, Ay and the Avenue of Sphinxes between Pylon $X$ and the Mut Precint at Karnak», BSGE 15, 31-38.

El-Khouly, A. \& MARTIN, G.T. (1987), Excavations in the Royal Necropolis at el-Amarna, Suppément ASAE 33, EI-Cairo.

Ertman, E. (1992), "Is there visual evidence for a king Nefertiti? ", Amarna Letters 2, 50-5.

FAIRMAN, H. (1972), "Tutankhamun and the End of the 18th Dynasty", Antiquity 46, 15-18.

GABOLDE, M. (1987), «Ay, Toutankhamon et les martelages de la stéle de restauration de Karnak (CG 34183)», BSGE 11, 37-61.

- (1992), "Baketaton fille de Kiya?», BSGE 16, 27-39.

GARDINER, A. (1928), "The Graffito from the Tomb of Pere», JEA 14, 10-11.

- (1953), "The Coronation of King Haremhab", JEA 39, 13-31.

Germer, R. Die Pflanzenmater aus dem Grab des Tutanchamun, Hildesheim 1989.

GrifFiths, J. G. (1991), The Divine Verdict. A Study of Divine Judggement in the Ancient Religios, Leiden.

GOHARY, J. (1992), Akhenaten's Sed-Festival at Karnak, Londres.

GUTERBOCK, H. "The Deeds of Suppiluliuma as told by his son, Mursili Il», JCS 10. 1956, 41-68; $75-98 ; 107-130$.

HABACHI, L. (1958), «God's Fathers and the Role they Plasyed in the History of the First Intermediate period", ASAE 55, 167-90.

-. (1969), Features of the Deification of Ramesses II, Glückstadt.

- (1979), "Unknown or little-known monuments of Tutankhamun and of his Viziers", Glimpses of Ancient Egypt. Studies in Honour H. W. Fairman, Londres 32-41. 
HARI, R. (1964), Horemheb et la reine Moutnedjemet, ou la fin d'une dynastie, Ginebra.

- (1985), New Kingdom. Amarna Period, Leiden.

HARRIS, J.R. (1974), «Nefernefruaten regnans», AO 36, 11-21.

- (1992), «Akhenaten and Nefernafruaten in the Tomb of Tut'ankhamun", After Tut'ankhamun, C. Reeves (Ed.), Londres, 55-72.

HARRISON, R. (1966), "An anatomical examination of the pharaonic remains purported to be Akhenaten", JEA 52, 95-119.

HaRRISON, R. \& ABDALLA, a (1972), «The Remains of Tutankhamun», Antiquity 46, 8-14.

HELCK, W. (1978), Die Beziehungen Ägyptens zur Vorderaisen, Berlín.

- (1981), «Nattkmin", LÄIV, Wiesbaden, 371-2.

HePPER, F. (1990), Pharaoh's Flowers: the Botanical Treasures of Tutankhamun, Londres.

KEMP, B. (1983-7), Amarna Reports I-V, Londres.

KITCHEN, K.A. (1962), Suppiluliumas and the Amarna Pharaohs, Liverpool.

KNUDTZON, J. (1908-15), Die El-Amarna Tafeln, Leipzig.

Krauss, R. (1978), Das Ende der Amarnazeit. Beiträge zur Geschichte und Chronologie des Neuen Reiches, HÄB 7, Hildesheim.

KRUCHTEN, J. M. (1981), Le Décret d'Horemheb. Traduction, commentaire épiraphique, philologique et institutionel, Brexelas.

LEGRAIN, G. (1907), "La grande stèle de Toutankhamonou à Karnak», RT 29, 162-173.

LEPROHON, R. (1985), "The Reign of Akhenaten seen throught the latter royal decrees", IFAO, $85,93-103$.

LiVERANI, N. (1983), «Political lexicon and political ideologies in the Amarna Letters», Berytus 31 , 41-56.

LoRTON, A. (1993), «The Instructions for Merikare and amarna Ideology», GM 134, 69-83.

MALEK, J. (1985), «The tomb-chapel of Hekamaestre-neheb at Northern Saqqara», SAK 12, 43-60.

- (1988), «Te Royal Butler Hori at Northern Saqqara», JEA 74, 125-136.

- (1992), «A meeting of the Old and New. Saqqara during the New Kingdom», Studies in Pharaonic Religion and Society in Honour of $J$. G. Griffiths, Londres, 57-76.

MANNICHE, L. (1975), "The wife of Bata", GM 18, 33-35.

Martin, G.T. (1989), The Memphite Tomb of Horemheb, Commander in chief of Tutankhamun 1: The Reliefs, Inscriptions and Commentary, EES 55, Londres.

- (1989a), The Royal Tomb at El-Amarna. The Rock Tombs of El-Amarna. Part VIIII. The Reliefs, inscriptions and architecture, EES 39, Londres.

MEYER, G. (1992), "Hurija und Pihururija", GM 126, 87-92.

MYSLIWIEC, K. (1979), "Amon, Atum and Aton: the evolution of Heliopolitan influences in Thebes", L'Egyptoloie en 1979, París, 285-289.

- (1990), «The Art of Amenhotep III. A link in a continouos evolution», The Art of Amenhotep III, Cleveland 16-25.

Murname, W. (1977), Ancient Egyptian Coregencies, SAOC 40, Chicago.

- (1990), The road to Kadesh. A historical interpretation of the Battle Reliefs of King Sety / at Karnak, SAOC 42, Chicago.

Ockinga, B. (1991), "The Tomb of Sennedjem at Awlad Azzaz», BACE 2, 81-9.

Pfluger, K. (1936), Haremhab und die Amarnazeit, Zwickau.

- (1991), The Hidden Tombs of Memphis. New discoveries from the time of Tutankhamun and Ramesses the Great, Londres.

Pérez LaRgacha, A. (1994), "Los Hiksos y Egipto. Transformaciones políticas, sociales, religiosas y económicas en el Imperio Nuevo», Aegyptiaca Complutensia 3, Luis A. García Moreno \& Pérez Largacha (Eds.).

- (1995), «Akhenatón, ¿pacifismo religioso?". Espacio, tiempo y forma, 7.

PHILLIPS, J. «Sculpture ateliers of Akhetaten», Amarna Letters 1, 1991, 31-40.

ReEves, C. (1982), "Akhenaten after all?», GM 54, 61-71.

- (1988), «New Light on Kiya from Texts in the Bristish Museum», JEA 74, 91-101.

- (1990), The Complete Tutankhamun, Londres.

- (1990a), Valley of the Kings. The Decline of a Royal Necropolis, Londres.

ReDFord, D. (1977), Akhenaten Temple Project I, Londres.

- (1984), Akhenaten. The Heretic King, Princeton.

- (1988), «Reliefs Scenes, mainly from the RWD-MNW», The Akhenaten Temple Project, vol. 2, Toronto, 1-12. 
- (1992), Egypt, Canaan and Israel in Historic times, Princeton.

RoBINS, G. (1981), «Hmt nsw wrt Meritaton», GM 52, 75-81.

RYAN, D. (1992), "Some observations concerning uninscribed tombs in the Valley of the Kings", After Tut'ankhamun, C. Reeves (Ed.), Londres, 21-7.

SAMson, J. (1978), Amarna, City of Akhenaten: Nefertiti as Pharaoh, Londres.

- (1982), «Akhenaten's coregent Ankhheprure-Nefernefruaten», GM 53, 51-54.

- (1982a), "Akhenaten's co-regent and successor», GM 57, 57-59.

SCHADEN, O. (1977), The God's Father Ay, Ann Arbor.

- (1984), «Clearance of the tomb of King Ay», JARCE 21, 39-64.

- (1992), "The God's Father Ay", Amarna Letters 2, 92-115.

SCHULMAN, A. (1964), «Excursus on the Military Officer Nakhtmin», JARCE 3, 124-126.

- (1979), "The Nubian War of Akhenatón», L'Egyptologie en 1979, París, 299-316.

- (1988), «Hittites, Helmets and Amarna: Akhenaten's first Hittite War", The Akhenaten Temple Project, vol. 2, Toronto, 53-80.

SeELE, K. «King Ay and the close of the Amarna Age», JNES 14, 1955, 170-80.

SeVERAL, M. (1972), "Reconsidering the Egyptian Empire in Palestine during the Amarna Period», PEQ 104, 123-133.

SMITH, H. (1976), The Fortress of Buhen. The Inscriptions, Londres.

Spalinger, A. (1979), «Egyptian-Hittite Relations at the close of the Amarna Period and some notes on Hittite Military Strategy in North Syria", Bulletin of the Egyptological Seminar, 1, 55 89.

SteWARd, H. (1976), Egyptian Stelae, reliefs and Paintings from the Petrie Collection, Parte 1, Warminster.

SüRENHAGEN, D. (1985), Paritätische Staatsverträe aus hethitischer Sicht, Pavia.

TOBIN, V. (1986), "Mythic Symbolism in the Amarna System», JSSEA 16, 5-18.

VANDERSLEYEN, C. (1992), "Royal Figures from Tut'ankhamun's Tomb: their Historical Usefulness", After Tut'ankhamun, C. Reeves (Ed.), Londres, 85-90.

VARILLE, A. (1940), "Toutankhamon, ets-il fils d'Aménophis III et de Satamon?", ASAE 40, 651657.

WeINSTEIN, J. (1973), Foundation Deposits in Ancient Egypt, Ann Arbor.

- (1981), "The Egyptian Empire in Palestine: a Reassessment», BASOR 241, 1-28.

WENTE, E. \& HARPIS, J. (1992), «Royal Mummies of the Eighteenth Dynasty: a Biologic and Egyptological approach», After Tut'ankhamun, C. Reeves (Ed.), Londres, 2-20. 